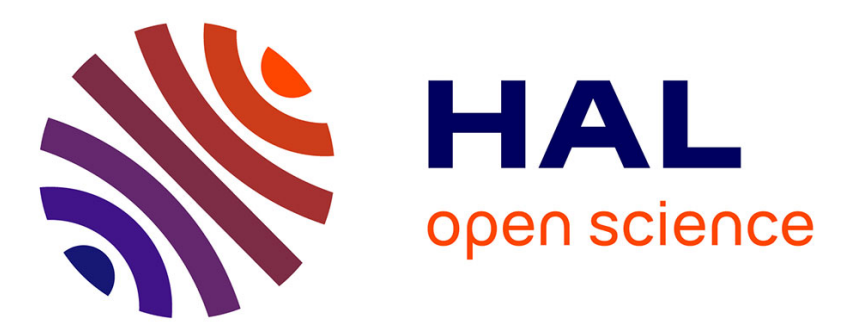

\title{
Stability, deformation and variability of granular fills composed of polyhedral particles
}

Quezada Juan Carlos, Breul Pierre, Saussine Gilles, Farhang Radjai

\section{To cite this version:}

Quezada Juan Carlos, Breul Pierre, Saussine Gilles, Farhang Radjai. Stability, deformation and variability of granular fills composed of polyhedral particles. Physical Review E : Statistical, Nonlinear, and Soft Matter Physics, 2012, 86, pp.031308. 10.1103/PhysRevE.86.031308 . hal-00689731

\section{HAL Id: hal-00689731 \\ https://hal.science/hal-00689731}

Submitted on 20 Apr 2012

HAL is a multi-disciplinary open access archive for the deposit and dissemination of scientific research documents, whether they are published or not. The documents may come from teaching and research institutions in France or abroad, or from public or private research centers.
L'archive ouverte pluridisciplinaire HAL, est destinée au dépôt et à la diffusion de documents scientifiques de niveau recherche, publiés ou non, émanant des établissements d'enseignement et de recherche français ou étrangers, des laboratoires publics ou privés. 


\title{
Stability, deformation and variability of granular fills composed of polyhedral particles
}

\author{
Juan Carlos Quezada, ${ }^{1, *}$ Pierre Breul, ${ }^{2}$ Gilles Saussine, ${ }^{1}$ and Farhang Radjai ${ }^{3}$ \\ ${ }^{1}$ SNCF Innovation and Research, Immeuble Lumire, \\ 40 Avenue des Terroirs de France,75611 Paris cedex 12, France. \\ ${ }^{2}$ Clermont Universit, Université Blaise Pascal, Institut Pascal, BP 10448, \\ F-63000 CLERMONT-FERRAND - CNRS, UMR 6602, IP, F-63171 Aubière, France. \\ ${ }^{3}$ LMGC, CNRS-Université Montpellier 2, Place Eugène Bataillon, 34095 Montpellier, France.
}

(Dated: April 13, 2012)

\begin{abstract}
By means of extensive contact dynamics simulations, we investigate the mechanical equilibrium and deformation of a granular material composed of irregular polyhedral particles confined between two horizontal frictional planes. We show that, as a consequence of mobilized wall-particle friction force at the top and bottom boundaries, the transient deformation induced by a constant vertical load is controlled by the aspect ratio (thickness over width) of the packing as well as the stress ratio. The transient deformation declines considerably for increasingly smaller aspect ratios and grows with the stress ratio. From the simulation data for a large number of independent configurations, we find that sample-to-sample fluctuations of the deformation have a broad distribution and they scale with the average deformation. We also analyze the evolution of particle connectivity during settlement and with the applied force. The face-face and edge-face contacts between polyhedral particles concentrate strong force chains with a growing proportion as a function of the applied force.
\end{abstract}

PACS numbers: 45.70.-n,61.43.-j,7.57.Gc

\section{INTRODUCTION}

Granular materials are widely used as filling materials in various applications due to their original complementary properties of flowability, shear strength and porosity [1]. Well-known examples are construction foundations, earthworks (dams, embankments, ...) and transport infrastructures (railway ballast, asphalt). A major concern in all such applications is the short- and long-term stability of the granular fills under variable loading conditions. Plastic deformations induced by static and dynamic overloads, such as train traffics in the case of railway ballast, or material degradation as a result of weathering, may lead to settlement and failure or damage to the structure $[2-5]$.

In absolute terms, a granular system is stable if small stress increments can be accommodated by small strains [6-9]. However, the particle-scale processes underlying this transition between consecutive equilibrium states are generally stochastic due to granular disorder [1014]. In constitutive modeling of the quasi-static behavior of granular materials, it is assumed that the strain and stress increments present a finite average and the fluctuations can be neglected. Elastoplastic models of soil behavior are based on this assumption with phenomenological internal variables or parameters pertaining to the packing structure. The plastic threshold is characterized by an internal friction angle and the flow rule by a dilatancy angle relating volume change to shear strain [14-17]. In such models, the stability coincides with hard-

\footnotetext{
${ }^{*}$ Electronic address: juan-carlos.quezada-guajardo@sncf.fr
}

ening behavior and the cumulative strains may be calculated from stress variations by means of the constitutive tensor for a representative volume element undergoing homogeneous deformation.

The stability and deformation of granular materials under complex boundary conditions are in general far less clear even when the rheological behavior is correctly captured. Failure may happen as a result of strain localization in shear bands or compaction bands [18]. Due to dilatancy, the material may also become unstable depending on how the boundary conditions are controlled $[7,8]$. For example, if the volume change is prohibited, a sufficiently loose sample will collapse when subjected to shear. This failure mode, known as static liquefaction, is homogeneous [6, 9].

Another important case is a granular material confined by frictional boundaries. This is a common and crucial part of the problem of stability in most applications and, in contrast to the fore-cited examples, it has a stabilizing effect on the granular assembly. The friction forces may be only partially mobilized depending on the dynamic processes and past deformations leading to the present state of a granular assembly. The importance of boundary friction is quite evident by remarking that a sandpile cannot be stable without friction at the basal plane. In experiments on granular flow on an inclined plane, it has been established that, due to friction with the plane, the angle at which the particles start to flow depends on the thickness of the granular bed [19, 20]. In the wellknown example of a silo, the wall friction governs stress transmission by fully compensating stress gradients due to particle weights [21-23]. A nontrivial effect of wall friction is to make depend the stress field inside the confined granular material on the width of the silo. Besides the case of silo, most past studies of wall friction have 
focused on the uniaxial compaction of granular materials in a mould [24-26], the effect of friction with a retaining wall [27], friction mobilization along an array of particles [28] and the effect of friction with side walls on granular flow $[29-32]$.

In this paper, we are interested in the equilibrium and deformation of a granular material confined between two horizontal frictional planes. The bottom plane is immobile whereas the top plane is mobile and subjected to a constant load. A uniform horizontal pressure is applied over the lateral boundaries of the sample of nearly cylindrical shape. The granular material is squeezed under the action of the imposed load and flows sideways by overcoming both the lateral confining pressure and friction forces with the top and bottom walls. As the deformation proceeds, the thickness of the packing declines and friction forces at the top and bottom walls increase until the deformation stops.

The stability and transient deformations are analyzed by means of extensive contact dynamics simulations with particles of irregular polyhedral shape for a large number of independent configurations, different thicknesses and values of the imposed load. In particular, we show that, due to wall friction, the total vertical settlement induced by the applied load depends both on the load and on the aspect ratio (thickness over width) of the packing. However, sample-to-sample fluctuations occur around the mean. From the data, we find that the distribution of transient deformations is broad and it scales with the mean deformation.

In the following, we first introduce in section II the numerical procedures including the simulation method, particle properties and preparation protocol. In section III, we illustrate the influence of various parameters such as the applied load, aspect ratio, particle inertia and loading history on the temporal behavior and total deformations. In section IV, a parametric investigation of total deformations and their sample-to-sample fluctuations is presented with aspect ratio and stress ratio as parameters, and compared with a simple model based on the analysis of the stresses inside the packing. Section V is devoted to the microstructure and its evolution. We conclude with a few remarks and summary of the most salient results of this work.

\section{NUMERICAL PROCEDURES}

In this section, we briefly introduce the contact dynamics (CD) method, as a discrete element method (DEM) for the simulation of granular materials, with polyhedral particles as well as the numerical procedures used for the preparation of the numerical samples.

\section{A. Contact dynamics method}

The simulations were carried out by means of the contact dynamics (CD) method with irregular polyhedral particles [33-38]. The CD method is a discrete element approach for the simulation of nonsmooth granular dynamics with contact laws expressing basically the mutual exclusions and dry friction between particles without introducing elastic or viscous regularization often used in explicit methods such as molecular dynamics [39-42] or the distinct element method introduced initially by Cundall and Strack [43]. Hence, this method is particularly adapted for the simulation of perfectly rigid particles. The nonsmoothness refers to various degrees of discontinuity in velocities arising in a system composed of rigid particles. In this method, the equations of motion for each particle are formulated as differential inclusions in which velocity jumps replace accelerations [44, 45]. The unilateral contact interactions and Coulomb friction law are treated as complementarity relations or set-valued contact laws.

The time-stepping scheme is implicit but requires explicit determination of the contact network at each time step. At each time step, all kinematic constraints implied by frictional contacts between particles are simultaneously taken into account, together with the equations of dynamics, in order to determine all velocities and contact forces in the system. This problem is solved by an iterative procedure pertaining to the non-linear Gauss-Seidel method. It consists of solving a single contact problem with other contact forces being treated as known, and iteratively updating the forces and velocities until a convergence criterion is fulfilled. The iterations in a time step are stopped when the calculated contact forces are stable with respect to the update procedure. It should be noted that, due to implicit time integration, the timestepping scheme is unconditionally stable.

The determination of the contact set for irregular polyhedral particles proceeds in three steps. First, a "bounding box" method is used to sort a list of neighboring particle pairs. Then, for each pair, the overlaps are calculated through a 3D extension of the "shadow overlap method" $[37,46]$. Several algorithms exist for overlap determination between convex polyhedra [47-50]. In the case of an overlap, the contact plane is determined by means of the intersection between the two particles. This detection procedure is fairly rapid and allows us to simulate large samples composed of polyhedral particles.

The contacts between polyhedral particles belong to different categories, namely face-face, edge-face, vertexface, edge-edge, vertex-vertex, vertex-edge. Face-face contacts are represented by three points, corresponding to three geometrical constraints, and thus will be referred below as triple contacts. The edge-face contacts are represented by two points and will be called double contacts. The edge-edge and vertex-face contacts are simple contacts and they are represented by a single point. The vertex-vertex and vertex-edge contacts are rare, but do 
occur from time to time in the course of evolution of the system. They are treated as a simple, double or triple contact on the basis of secondary criteria. In the iterative procedure of determination of the contact forces and velocities, the points representing the contacts between two particles are treated as independent points but the resultant force of the calculated point forces are attributed to the contact with its application point located on the contact plane.

For our simulations, we used the LMGC90 which is a multipurpose software developed in Montpellier, capable of modeling a collection of deformable or undeformable particles of various shapes by different algorithms [37], see www.lmgc.univ-montp2.fr/ dubois/LMGC90. Video samples of the simulations analyzed in this paper can be found at www.cgp-gateway.org/ref014.

\section{B. Sample preparation}

The numerical samples are composed of rigid polyhedral particles taken from a library of 1000 digitalized ballast particles[63]. Each particle has at most 70 faces and 37 vertices and at least 12 faces and 8 vertices. Fig. 1(a) shows examples of the polyhedral particles used in the simulations. The size of a particle is defined as two times the largest distance between the barycenter and the vertices of the particle, to which we will refer as the "diameter" of the particle. The particle sizes vary between $25 \mathrm{~mm}$ and $50 \mathrm{~mm}$ with $50 \%$ of diameter $25 \mathrm{~mm}$, $34 \%$ of diameter $37.5 \mathrm{~mm}$ and $16 \%$ of diameter $50 \mathrm{~mm}$. The mean particle diameter is $\langle d\rangle=37.5 \mathrm{~mm}$. The bulk density of the particles is $2700 \mathrm{~kg} \mathrm{~m}^{-3}$. The coefficient of friction between the particles is $\mu=0.8$ for all samples. The normal and tangential coefficients of restitution are set to zero. The value of coefficient of restitution has no significant effect on the dynamics of dense granular systems where frictional dissipation prevails and collisions are basically of multi-contact nature due to the presence of a dense contact network.

The preparation protocol consists in pouring first the particles into a cylindrical box with zero particle-wall friction. A rigid wall of mass $m_{w} \simeq 16 \mathrm{~kg}$ is placed on top of the sample. Then, the cylinder is removed and replaced by a radial confining pressure of $\sigma_{R}=80$ $\mathrm{kPa}$ applied by a uniform distribution of radial forces over the outermost particles located on the periphery of the cylinder while keeping the bottom wall fixed. The sample obtained by this procedure is subjected to vibrations of small amplitude by applying a vertical sinusoidal displacement on the top wall. The vibrations last for about $0.4 \mathrm{~s}$ with a frequency of $10 \mathrm{~Hz}$. The vibrations remove the irregularities at the interface between the block and the top layer of particles. Finally, the vibration is stopped and the sample is allowed to relax to equilibrium.

We prepared different samples with the same radius $R \simeq 0.35 \mathrm{~m}$ but with different heights $H$ depending on the number of particles. By changing the number of par-

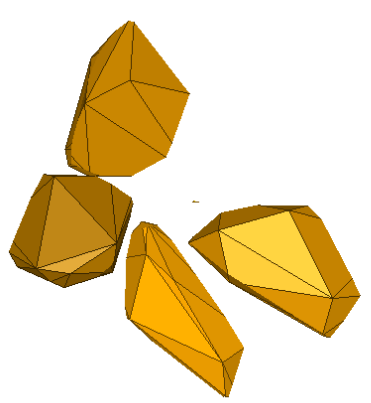

(a)

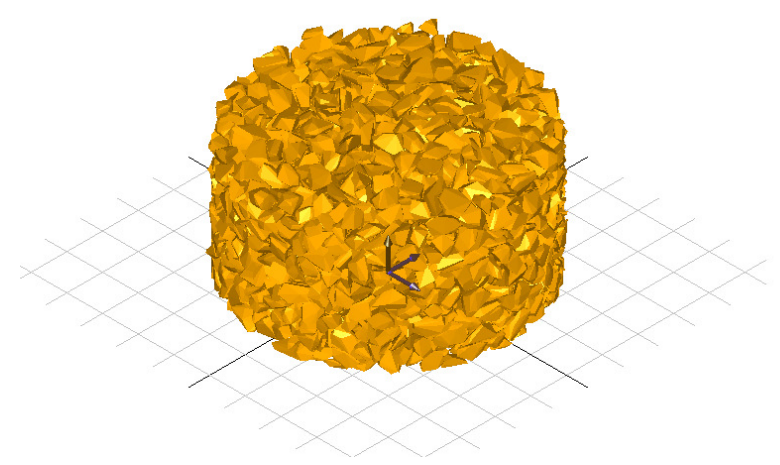

(b)

FIG. 1: (a) Four examples of polyhedral particles used in the simulations. (b) Snapshot of a numerical sample.

ticles from $N_{p}=1600$ to $N_{p}=3200$, we get different samples with aspect ratios $\alpha=H /(2 R)$ varying from 0.4 to 0.7 . As we shall see below, a detailed statistical analysis is performed for the samples with $\alpha=0.5$ (containing 2700 particles) by generating 32 independent configurations. The applied protocol results in packing fractions $\rho$ for the 32 samples varying in the range $[0.610,0.626]$. Fig. 1(b) shows one snapshot of a numerical sample.

In a system prepared by the above procedure, there is no lateral wall and the radial pressure is supported by the outmost particles, which behave in this way as a flexible "membrane" confining the sample. This is very similar to the usual triaxial compression tests performed on soil samples but with the major difference that in a triaxial device the radial displacements of the particles are strongly constrained by the presence of a real membrane fixed to the top and bottom plates. In our system, we impose a large friction coefficient $\mu_{w}=1$ at the contact with the top and bottom walls. This corresponds to the condition of "rough walls" that prevents the particles in contact with the top and bottom walls to slide [27]. Hence, the stability of the sample is controlled both by the bulk friction and the friction with the top and bottom walls.

The transient deformations of the numerical samples are studied by applying an force $F$ on the top wall. 500 different values of $F$ varying from $1 \mathrm{kN}$ to $120 \mathrm{kN}$ are applied to each sample in order to obtain both the mean value and variability of the deformation. Given the initial area of the sample $S=\pi R^{2} \simeq 0.38 \mathrm{~m}^{2}$, the vertical 


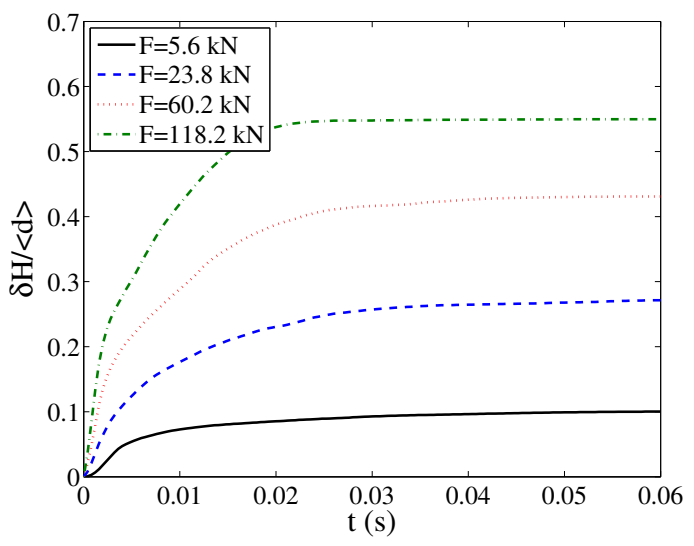

FIG. 2: Settlement of a sample of aspect ratio $\alpha=0.5$ subjected to different values of the vertical force $F$ as a function of time.

stress $\sigma_{H}=F / S$ supported by the sample varies in the range $[2,315] \mathrm{kPa}$. The time step was $4 \cdot 10^{-4} s$ in all simulations and at most 150 time steps were needed for a full stabilization in the case of the longest transient. The CPU time was $2.10^{-3} s$ per particle and per time step on a Dell computer of speed $3.16 \mathrm{GHz}$.

\section{TRANSIENT DEFORMATIONS}

In this section, we would like to illustrate the sensitivity of the vertical deformation to different system parameters. This will allow us to define the relevant parameters for a quantitative description of the behavior in the following sections. The downward cumulative displacement of the top wall is denoted by $\delta H$ and normalized by the mean particle diameter $\langle d\rangle$.

Figure 2 displays $\delta H$ as a function of time in a sample of aspect ratio $\alpha=0.5$ corresponding to a height $H=2 \alpha R \simeq 0.35 \mathrm{~m}$, subjected to different values of the force $F$. The evolution seems to occur in three stages with a fast increase of $\delta H$ at the beginning and a slower evolution at intermediate times followed by a longer phase with small change of $\delta H$. As expected, the total settlement $\Delta H=\int_{0}^{\infty} \delta H(t) d t$ increases with $F$. The displacements are typically below one particle diameter. The vertical deformation for a displacement of one mean particle diameter is $\langle d\rangle / H \simeq 0.11$.

The time evolution of $\delta H$ reflects the dynamic particle rearrangements induced by sudden application of the load. As a result of particle inertia and collisions, the normal force $F_{b}$ at the basal plane increases largely beyond the applied force $F$ as shown in Fig. 3. $F_{b}$ grows in a short time lag to a peak two times above $F$. This time interval coincides with the initial rapid increase of $\delta H$. The kinetic energy gained by the particles is not fully dissipated by this collective shock of the sample with the bottom wall since $F_{b}$ takes much more time to relax from its peak value towards the force $F$, where the particles

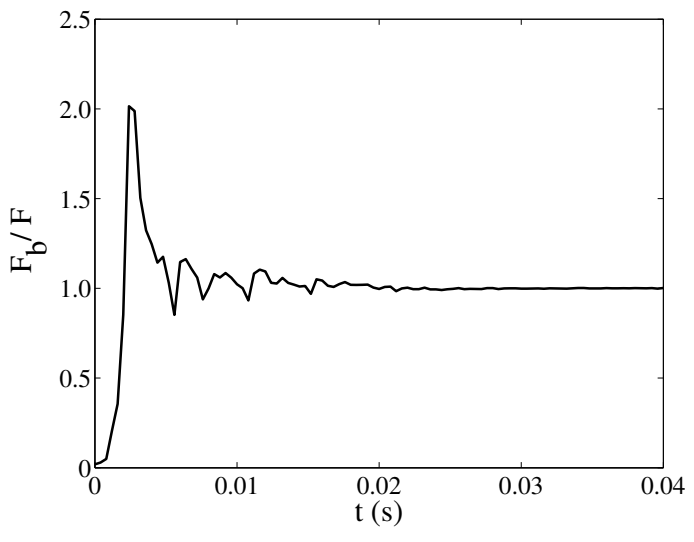

FIG. 3: Time evolution of the normal force $F_{b}$ at the basal plane following the application of a constant force $F=60 \mathrm{kN}$ on the top plane.

jam in a new state of static equilibrium. Comparing the times series of $\delta H$ and $F_{b}$, we observe that the particle rearrangements from the peak to the end of relaxation contribute as much to the settlement of the packing as the highly dynamic evolution from the application of the force to the peak.

The characteristic time $\Delta t$ of the dynamic transient is obtained by dimensional analysis from the natural units of the system: particle mass $m$, average pressure $p$ and particle size $\langle d\rangle$. We get

$$
\Delta t=\left(\frac{m}{p\langle d\rangle}\right)^{1 / 2}
$$

This time may be interpreted as the time for a particle of mass $m$ to fall a distance equal to its own diameter under the action of a force equal to $p d^{2}$ [20]. For an average pressure of the order of the lateral pressure $\sigma_{R}=80$ $\mathrm{kPa}$, we get $\Delta t \simeq 0.005 \mathrm{~s}$, which is the right order of magnitude of the observed relaxation time according to Figs. 2 and 3.

Clearly, as a consequence of the application of a finite force on an initially stable packing, the dynamics plays an essential role in the total settlement $\Delta H$. In order to illustrate this effect, we applied the same total force $F=60 \mathrm{kN}$ on a sample in a single step, in two successive steps of $30 \mathrm{kN}$, in three steps of $20 \mathrm{kN}$ or in six steps of $10 \mathrm{kN}$ by allowing the sample to relax to equilibrium in each step. The time evolution of $\delta H$ is shown in Fig. 4 for these four simulations. The largest settlement is reached for the application of the total force in a single step, and the total settlement declines when the number of intermediate steps increases. The lowest settlement is obtained by quasi-static compression, which corresponds to a large number of small force increments.

An important feature of step-wise compression test, as observed in Fig. 4, is that most of deformation occurs in the first step. The partial settlement in the next steps is smaller and keeps nearly the same magnitude in 


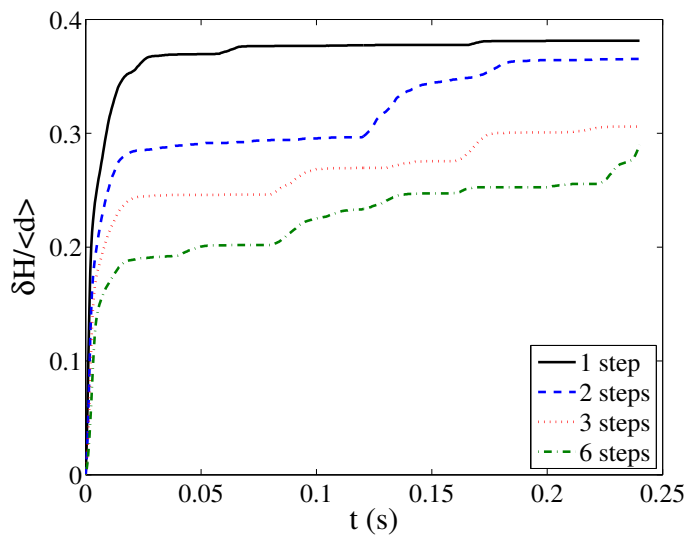

FIG. 4: Settlement of a sample subjected to a total vertical force of $60 \mathrm{kN}$ applied in a single step, two steps, three steps or six steps.

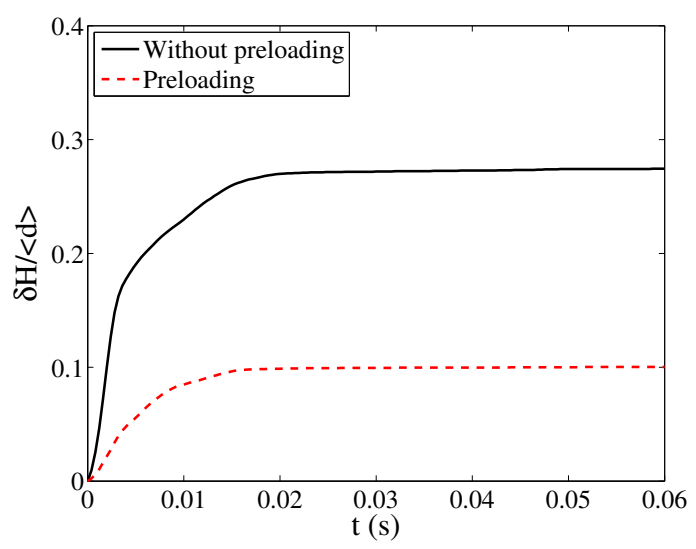

FIG. 5: Settlement of a sample subjected to a total vertical force of $60 \mathrm{kN}$ in two samples with and without consolidation.

each step. This means that the microstructure is modified by the application of the first load. This effect of preloading is illustrated in Fig. 5 where the time evolution of $\delta H$ is plotted for $F=60 \mathrm{kN}$ in a sample without preloading and in the same sample preloaded by the application of a force $F=3.6 \mathrm{kN}$ and allowed to relax. The preload is removed after the relaxation. We see that the evolution is smoother in a sample consolidated by the application of the preload and the settlement is considerably lower. The effect of preloading is similar to that of primary consolidation in soils [15]. Although the boundary conditions are very different here from those used for the compaction of soils, in both cases the application of a preload leads to irreversible reorganization of the contact network enhancing its strength in response to a secondary compression in the same direction [50, 51].

Due to friction with the top and bottom walls, the settlement for a given applied load crucially depends on the aspect ratio $\alpha$. Fig. 6 shows the time evolution of $\delta H$ in response to the application of a force $F=60 \mathrm{kN}$ for several samples of different aspect ratios. We see that

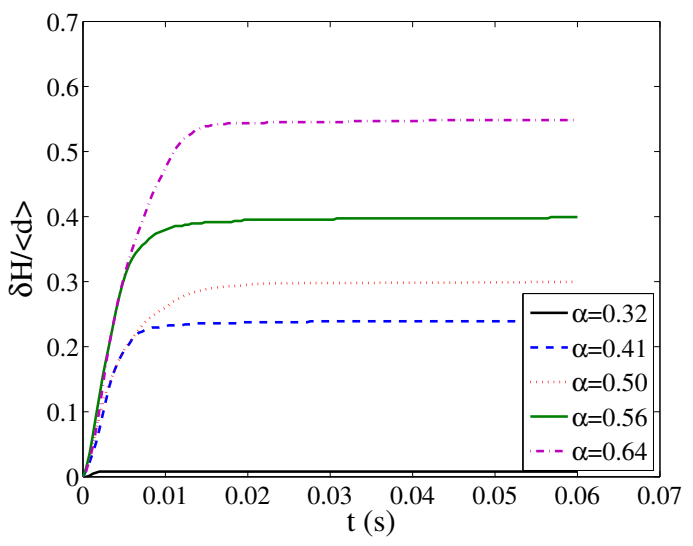

FIG. 6: Time evolution of vertical deformation for different samples of different aspect ratios $\alpha$ but the same applied load $F=60 k N$.

larger aspect ratios imply larger settlement. The total settlement is negligibly small for $\alpha=0.32$.

To analyze the role of aspect ratio, let us consider the condition of mechanical equilibrium by assuming perfect axial symmetry of the sample and friction at the top and bottom walls. The axial symmetry implies $\sigma_{r \theta}=0$. It is obvious that, as a result of cylindrical geometry and radial friction, the stress state is not homogeneous. As a simplifying approximation, we assume that the radial stress component $\sigma_{r r}$ depends only on the radial distance $r$ from the axis of the sample; see Fig. 7 . Therefore the condition of force balance for a volume element enclosed between two cylindrical surfaces of radii $r$ and $r+\delta r$ reads

$$
2 T(r)+2 \pi(r+\delta r) H \sigma_{r r}(r+\delta r)-2 \pi r H \sigma_{r r}(r)=0
$$

where $T(r)$ is the friction force exerted by the top and bottom walls against a radial deformation of the sample.

To solve equation (2), we further assume a linear relation every where inside the packing between the vertical and radial stress components:

$$
\sigma_{z z}=k \sigma_{r r} .
$$

This assumption underlies also the Janssen model and yields a correct prediction of stress transmission in a silo $[22,52]$. The values of the coefficient $k$ are bounded between $k_{p}$ and $k_{a}=k_{p}^{-1}$, corresponding to the so-called limit Rankine passive and active states, with [27]

$$
k_{a}=\frac{1+\sin \varphi}{1-\sin \varphi}
$$

where $\varphi$ is the internal angle of friction. We recall that the major principal stress $\sigma_{1}$ coincides with vertical stress $\sigma_{H}$ in the active state and with radial stress $\sigma_{R}$ in the passive state.

Assuming that, at incipient flow, the friction force $T(r)$ at the walls is fully mobilized, we have

$$
T(r)=2 \pi r \delta r \tau(r)=2 \pi r \delta r \mu_{w} \sigma_{z z}(r),
$$




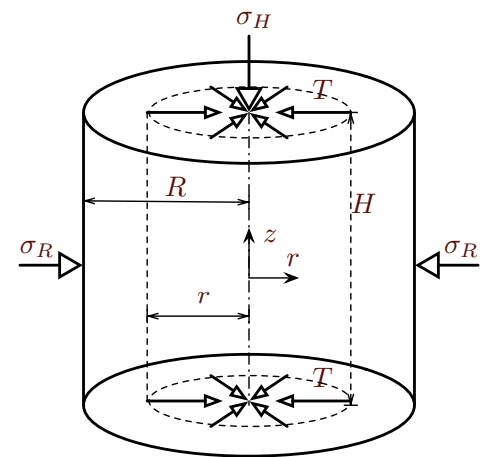

FIG. 7: Geometry and boundary conditions of the granular system in the presence of wall friction forces $T$.

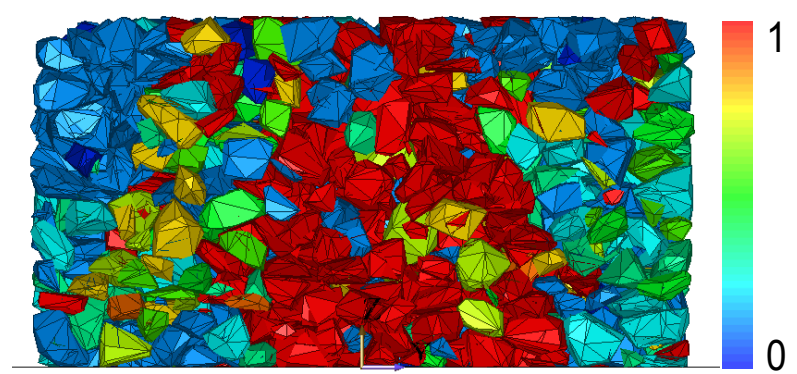

FIG. 8: A snapshot of particle stresses in a vertical section of the sample passing through the axis of symmetry.

where $\mu_{w}$ is the coefficient of friction at the walls. For a nearly rough wall, which is the case in our system with $\mu_{w}=1$, the particles do not slide at the walls and the friction mobilization is bounded by the internal friction coefficient, so that it is physically plausible to set $\mu_{w}=$ $\tan \varphi$.

Equations (2), (3) and (5), together with the boundary condition $\sigma_{r r}(r=R)=\sigma_{R}$, yield

$$
\frac{\sigma_{r r}(r)}{\sigma_{R}}=\frac{R}{r} \exp \left\{\frac{\mu_{w} k}{\alpha}\left(1-\frac{r}{R}\right)\right\} .
$$

According to this equation, the stresses diverge at the center as $1 / r$ and decline exponentially to $\sigma_{R}$ at the approach of the periphery of the sample. In practice, it is more convenient to consider the resultant force $f_{r}(r)=2 \pi r H \sigma_{r r}(r)$, which is not singular at the center and varies as

$$
f_{r}(r)=f_{R} \exp \left\{\frac{\mu_{w} k}{\alpha}\left(1-\frac{r}{R}\right)\right\},
$$

where $f_{R}=2 \pi R H \sigma_{R}$.

For the friction mobilization at the walls, we should distinguish the active state where the particles tend to flow outward and therefore the radial friction forces exerted by the top and bottom walls on the sample are oriented radially inward, from the passive state where the particles tend to flow inward (due to the action of the lateral stress) and therefore the radial friction forces are oriented radially outward. With our sign conventions, the inward friction force behaves as a compressive force and should be counted positive whereas the outward friction force is negative and we should replace $\mu_{w}$ by $-\mu_{w}$ in equations (7) and (6). Hence, the radial force declines in the active state from the center $(r=0)$ towards the periphery with $f_{r}(0)=f_{R} \exp \left(\frac{\mu_{w} k_{a}}{\alpha}\right)$ and it declines in the passive state from the periphery $(r=R)$ towards the center with $f_{r}(0)=f_{R} \exp \left(-\frac{\mu_{w} k_{p}}{\alpha}\right)$. Fig. 8 displays a map of "particle stresses", i.e. the average stresses supported by the particles due to the forces exerted by their contact neighbors, for a packing in the active state. As expected, the strongest force chains occur at the center of the sample and decline in intensity towards the periphery.

Equation (6) can now be used with the condition $\int_{0}^{R} \sigma_{z z}(r)(2 \pi r) d r=\pi R^{2} \sigma_{H}$, to relate the stress ratio to the aspect ratio. We get

$$
\eta \equiv \frac{\sigma_{H}}{\sigma_{R}}=2 \frac{\alpha}{\mu_{w}}\left\{\exp \left(\frac{\mu_{w}}{\alpha} k\right)-1\right\}
$$

This is an interesting relationship as it shows that, under the assumption of friction mobilization at the walls, the stress ratio $\eta$ is the relevant parameter for the stress state and its limit values depend on the aspect ratio $\alpha$. $\eta$ tends to $2 k$ in the limits of $\mu_{w} / \alpha \rightarrow 0$ and increases without bound when $\mu_{w} / \alpha \rightarrow \infty$. The effect of friction is amplified by the exponential factor and it leads to increasingly large values of $\eta$. In the limit active state, $k$ should be replaced by $k_{a}$ whereas in the limit passive state one should set $k=k_{p}$ and $\mu_{w}$ should be replaced by $-\mu_{w}$ :

$$
\begin{aligned}
\eta_{\text {active }} & =2 \frac{\alpha}{\mu_{w}}\left\{\exp \left(\frac{\mu_{w}}{\alpha} k_{a}\right)-1\right\} \\
\eta_{\text {passive }} & =2 \frac{\alpha}{\mu_{w}}\left\{1-\exp \left(-\frac{\mu_{w}}{\alpha} k_{p}\right)\right\}
\end{aligned}
$$

For a system of aspect ratio $\alpha=0.5$ and internal friction angle $34^{\circ}$, which is the case of the ballast material used in our simulations [50], we get $\eta_{\text {active }} \simeq 160$, which is far higher than $2 k_{a} \simeq 7$ for non-frictional walls, and $\eta_{\text {passive }} \simeq 0.47$, which is slightly below $2 k_{p} \simeq 0.57$. The reason is that the effective values of $k$ and $\mu_{w}$ starting from an initial state prepared by vibrations or consolidated by the application of an preload, reflect the mobilization of internal friction (for $k$ ) and wall friction (for $\mu_{w}$ ). The packing has a hardening behavior due to the evolution of the microstructure as increasingly larger loads are applied, and in our simple model of stress transmission the hardening is basically represented by the increase of the effective values of $k$ and $\mu_{w}$. On the other hand, the plastic deformation of our system is also partially governed by finite size effects that, given the limited number of particles, can not be avoided in 3D simulations with particles of complex shape. 


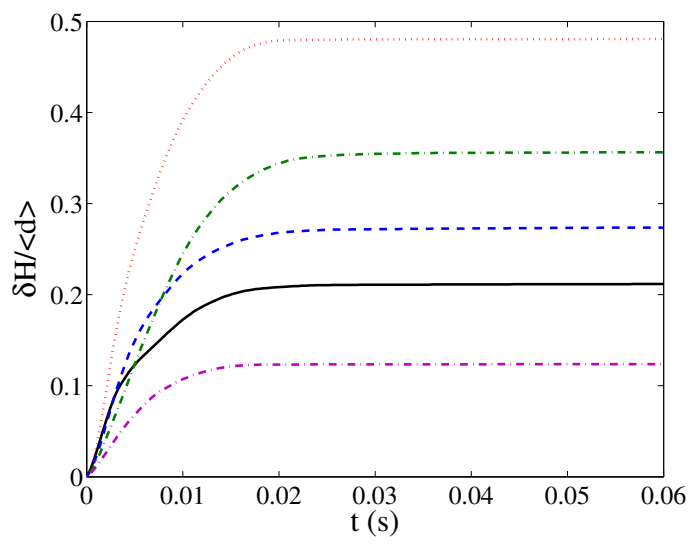

FIG. 9: Time evolution of vertical deformation in four samples for the same value of $\eta$ and aspect ratio $\alpha$ but different initial configurations.

Another consequence of finite size effects for our system is the fluctuation of deformations in independent samples prepared identically. Fig. 9 displays the time evolution of $\delta H$ for five different samples of the same aspect ratio $\alpha$ prepared independently, but according to the same protocol, and subjected to the same vertical load or stress ratio $\eta$ applied in a single step. We see that the transient deformations take nearly the same time but lead to different settlements. This rather large variability in the settlement reflects the stochastic nature of the deformation process which amplifies small differences in the initial packing fraction as well as subtle details of the contact network.

In the next section, we present a parametric study of the total vertical deformation $\Delta H$ and its statistical fluctuations as a function of $\alpha$ and $\eta$ for an ensemble of identically prepared and consolidated samples.

\section{TOTAL DEFORMATION AND ITS VARIABILITY}

Because of sample-to-sample fluctuations and the influence of consolidation and particle inertia, we need a large number of simulations in order to be able to quantify the dependence of the total deformation $\Delta H$ with respect to the applied stress ratio $\eta$ and aspect ratio $\alpha$. The simulations are performed for 9 different aspect ratios varying from $\alpha=0.36$ to $\alpha=0.64$. For each value of $\alpha$, we generate 32 independent samples by the procedure described in section II. A consolidation stress $\sigma_{H}=40$ $\mathrm{kPa}$ (corresponding to $\eta=0.5$ ) is applied to all samples and removed after relaxation. Each sample is subjected to 500 different values of the vertical force with $\eta$ varying in the range $[2,315]$. The vertical force in all simulations is applied in a single step so that the dynamic effects are systematically present in the resulting settlement. The data are analyzed by considering both the ensemble average of the vertical deformation over the 32 independent

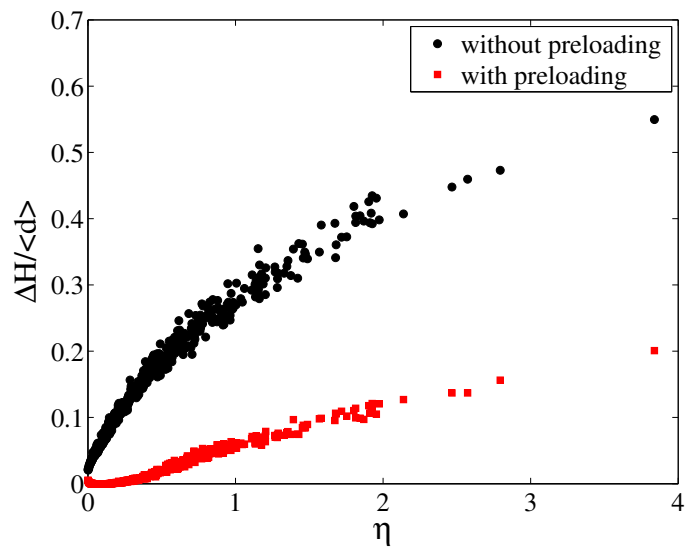

FIG. 10: Total settlement as a function of $\eta$ in a sample of aspect ratio $\alpha=0.5$ for the initial configuration (before preloading) and preloaded configuration.

samples for given $\eta$ and $\alpha$ and the variability around the average deformation.

The consolidation stress is large enough to predispose the granular packing to stress increments applied in the same direction. As shown in section III, the dynamic effects are reduced by preloading, and consecutive loads of the same magnitude produce equal settlements. Due to the plastification of the packing in the consolidation step, the application of a stress ratio $\eta$ below 0.5 will produce tiny deformations, as shown in Fig. 10 for a packing with aspect ratio $\alpha=0.5$. In the whole range, the consolidated packing undergoes lower settlement $\Delta H$, but the difference is most remarkable for $\eta<0.5$. $\Delta H$ increases with $\eta$ at a lower rate in the consolidated samples than unconsolidated ones. All the data presented in the following concern only the consolidated samples.

Figure 11 shows the total averaged settlement $\Delta H$ as a function of $\eta$ for all aspect ratios. $\Delta H$ is an increasing function of $\eta$ at a rate that increases with $\alpha$. Apart from the weak values of $\eta$ (close to the consolidation stress ratio 0.5 ), where the deformation is equally low for all aspect ratios, and largest value of $\eta$ where the data seem to curve slightly down, $\Delta H$ is nearly linear as a function of $\eta$. The same settlement data are represented in Fig. 12 as a grey level map in the parameter space $[\alpha, \eta]$. The isovalue lines of $\Delta H /\langle d\rangle$ are plotted for the data smoothed out over the close neighborhood of each data point. The map shows how the same amount of settlement may occur for a combination of different aspect ratios and stress ratios. The settlement is negligibly small for $\alpha<0.4$ and $\eta<0.5$ unless for very high stress ratios or large aspect ratios, respectively.

Since $\alpha$ is a control parameter for deformation, it is also interesting to use the variations $\delta \alpha$ of the aspect ratio as strain variable. Fig. 11 indicates that the rate $\delta \alpha / \delta \eta \simeq(1 / 2 R)(-\delta H / \delta \eta)$ is a well-defined quantity that can be extracted from the data by taking the mean slope of each plot. Fig. 13 shows the rate $-\delta \alpha / \delta \eta$ as a function of $\alpha$. It increases from 0.003 for $\alpha \simeq 0.35$ to 0.015 for 


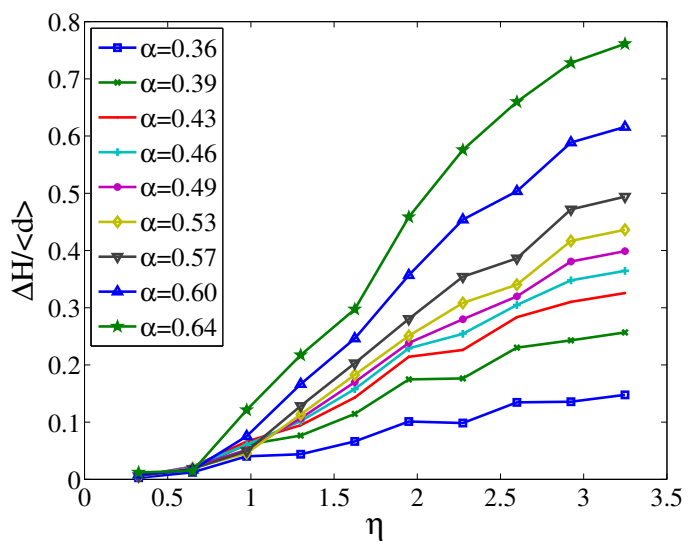

FIG. 11: Total normalized settlement as a function of stress ratio for different aspect ratios.

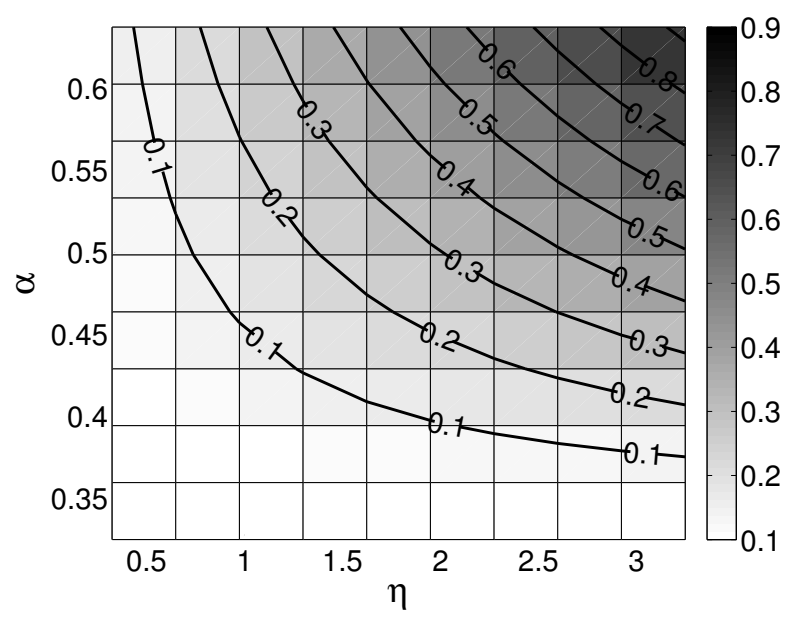

FIG. 12: Grey-level map of settlements as a function of aspect ratio $\alpha$ and stress ratio $\eta$. The full lines represent iso-values of the total settlement $\Delta H /\langle d\rangle$.

$\alpha \simeq 0.65$ with a change of trend around $\alpha=0.5$.

A theoretical dependence of $\delta \alpha / \delta \eta$ on $\alpha$ may also be obtained by deriving equation (8) for the active states with respect to $\alpha$. We get

$$
-\frac{\delta \alpha}{\delta \eta}=\frac{\mu_{w}}{2}\left\{1+\left(\frac{\mu_{w} k}{\alpha}-1\right) \exp \left(\frac{\mu_{w} k}{\alpha}\right)\right\}^{-1} .
$$

This equation yields an exponential increase as a function of $\alpha$ with the right order of magnitude of the rate by setting $k=2.2$ and $\mu_{w}=\tan \varphi \simeq 0.67$, as shown in Fig. 13. This model can not fully capture the observed behavior. However, it remains an interesting point of departure as it coveys an interesting physical picture of the mechanism of settlement: The settlement is triggered by an increment of stress ratio $\delta \eta$. The friction at the top and bottom walls increases due to the increase of the contact surface $\pi R^{2}$ with the decrease of aspect ratio. The settlement stops when $\delta \eta$ is balanced by the friction force. This frictional feedback implies an exponential decrease

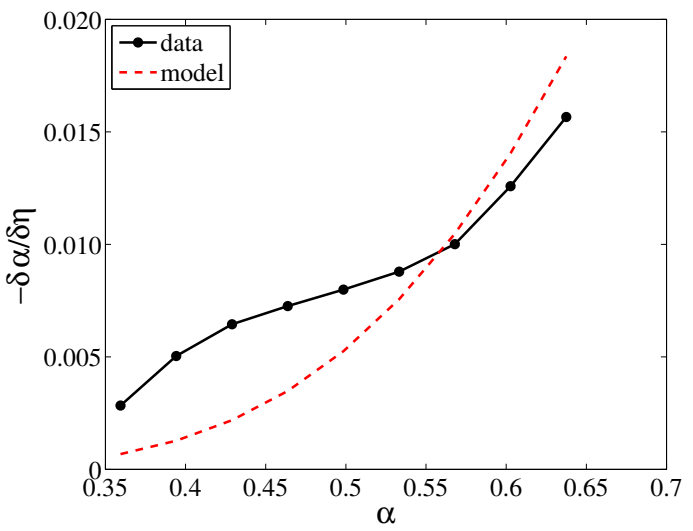

FIG. 13: Rate of change of the aspect ratio $\alpha$ with respect to stress ratio $\eta$ as a function of $\alpha$ from the simulation data (symbols) and as predicted by equation (11) (dashed line).

of the settlement rate for decreasing $\alpha$. Since the suggested value of $k$ is below the theoretical value $k_{a}=3.53$ for the limit active state, the process seems to be governed by a partial mobilization of the internal friction.

We turn now to the fluctuations around the average values of the settlements as a function of $\eta$. Fig. 14(a) shows the standard deviation $S$ of the settlement normalized by the mean particle diameter calculated from 32 independent samples of aspect ratio $\alpha=0.5$ for each value of $\eta$ with and without a consolidation step. We see that without consolidation $S$ rises with $\eta$ at a rate that is much faster in the range $\eta<0.5$ whereas with consolidation a negligibly weak variation occurs in this range. Its order of magnitude for larger values of $\eta$ becomes comparable to the average settlement. To compare $S$ with the average deformation, we have plotted the coefficient of variation, defined by the ratio $C_{v}=S / \Delta H$ of $S$ and the average settlement $\Delta H$, as a function of $\eta$ in Fig. 14(b). The coefficient of variation is high at low values of $\eta$ but declines rapidly in the range $\eta \in[0,0.1]$ and then continues to decrease at a lower rate. This high variability at low stress ratios represents a ratio of two small numbers (both the settlement and its standard variation) but it is a signature of the stochastic processes governing the particle displacements when the external forcing is too weak to impose collective deformations compared to single-particle events.

Figure 15 shows the probability density function of the settlements $\Delta H$ for all samples where the settlements are simply normalized by the mean settlement $\langle\Delta H\rangle$ for each value of $\eta$. Remarkably, in spite of the high dispersion of the values of $\Delta H$, the data collapse on a single distribution due to normalization by the mean settlement. The distribution can be approximately fit to a decreasing exponential function

$$
P(\Delta H) \propto \exp \left(-\gamma \frac{\Delta H}{\langle\Delta H\rangle}\right)
$$

with $\gamma \simeq 0.8$. Although small settlements are most fre- 

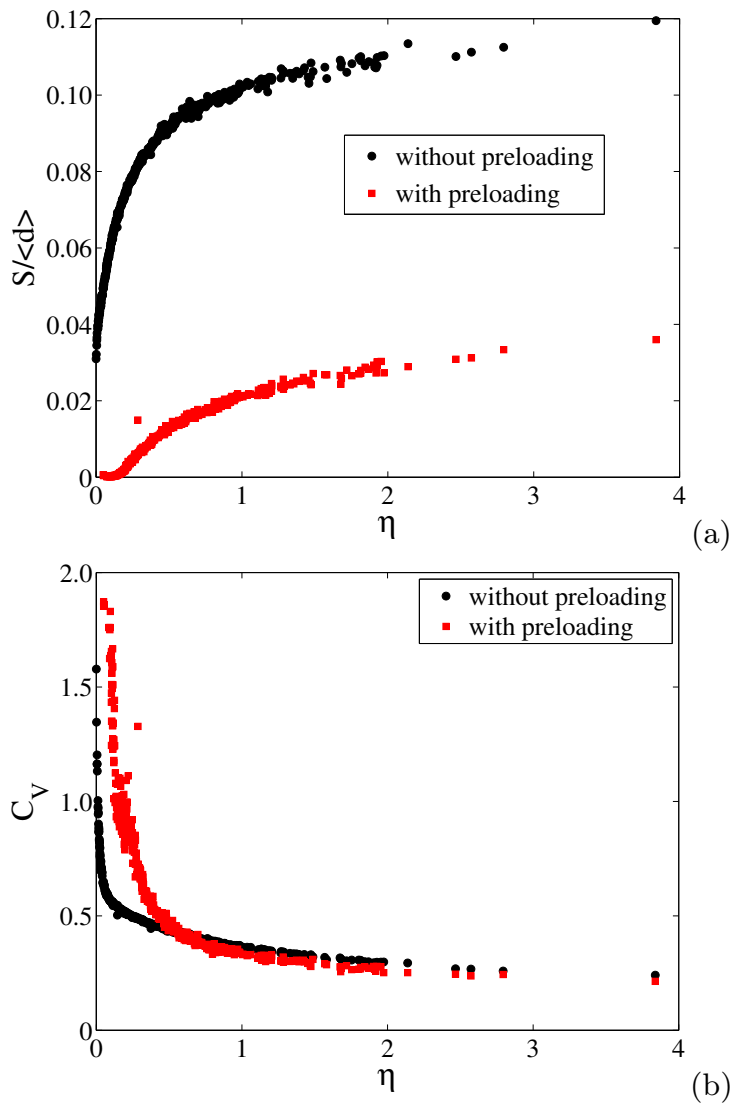

(b)

FIG. 14: (a) Standard deviation $S$ of settlements calculated for 32 independent samples for each value of stress ratio $\eta$ with and without preloading; (b) Coefficient of variation as a function of $\eta$.

quent, the exponential fall-off indicates that large settlements may occur with a significant probability. An exponential distribution of settlements means that the fluctuations are an intrinsic part of the deformation process and controlled by the granular disorder for all levels of loading. Equation (12) together with the map of average settlements in Fig. 12 provide a detailed information both on the mean dependence of settlement with respect to aspect ratio and stress ratio and on its sampleto-sample variability.

\section{FABRIC VARIABLES}

The fabric, i.e. the spatial organization of particles and the topology of the force-bearing contact network, encodes most of the past history of loading [50, 53-56]. In this section, we briefly investigate the evolution of several fabric variables during the settlement as a function of the applied stress. These are the mean coordination number $z$, the proportions $K_{s}, K_{d}$ and $K_{t}$ of simple, double and triple contacts, respectively, as well as the mean force carried by each type of contact.

Figure 16(a) displays a typical example of the evolution

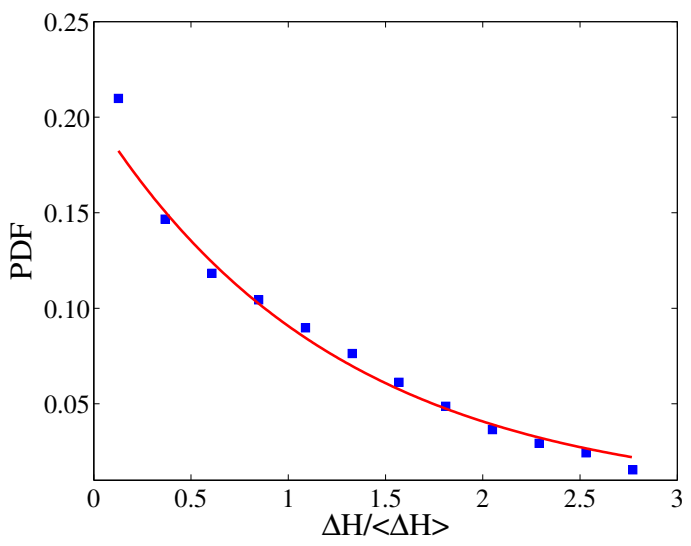

FIG. 15: Probability density function of settlements $\Delta H$ in all samples of aspect ratio $\alpha=0.5$ normalized by the mean settlement for each value of stress ratio (symbols). The full line is an approximate exponential fit $P(\Delta H) \propto \exp (-\gamma \Delta H /\langle\Delta H\rangle)$ with $\gamma \simeq 0.8$.

of $z$ as a function of time in a sample after the application of a stress ratio $\eta=2.5$. The initial fast drop of $z$ reflects the opening of a large number of contacts belonging to the weak contact network as a result of particle displacements, a feature usually observed at the beginning of plastic deformation $[14,57,58]$. The second peak is the signature of collective dynamic compaction of the sample corresponding to the first peak of the force observed in Fig. 3 followed by a slight decompation and decrease of $z$. The relaxation continues afterwards at a lower rate with a gradual increase of $z$ towards a constant level comparable to its initial value.

The time evolution is shown in Fig. 16(b) separately for $K_{s}, K_{d}$ and $K_{t}$ in the same sample. We observe the same dynamic effects as for $z$. But, the main effect of settlement is to reduce the fraction of simple contacts and to increase considerably that of double and triple contacts. The edge-face and face-face contacts allow for stable column-like structures, and their growth in the sample is a signature of hardening and enhanced stability of the packing although the proportion of simple contacts prevails at all times. The double and triple contacts capture strong force chains and concentrate stresses. Fig. 16(c) shows the mean normal forces $f_{s}, f_{d}$ and $f_{t}$ carried by simple, double and triple contacts as a function of time. The triple contacts, despite their weak presence in the packing, carry the largest forces, with a mean value of nearly 1.5 times the mean force whereas the simple contacts carry the lowest forces with an average below the mean. The double contacts carry a mean force of the order of the mean force in the packing.

The above trends of fabric evolution are increasingly reinforced as the stress ratio increases. For example, Fig. 17 shows the evolution of the proportions of different types of contact at the end of settlement as a function of $\eta . K_{s}$ declines systematically with $\eta$ whereas $K_{d}$ and $K_{t}$ increase. We also find that the mean forces carried 


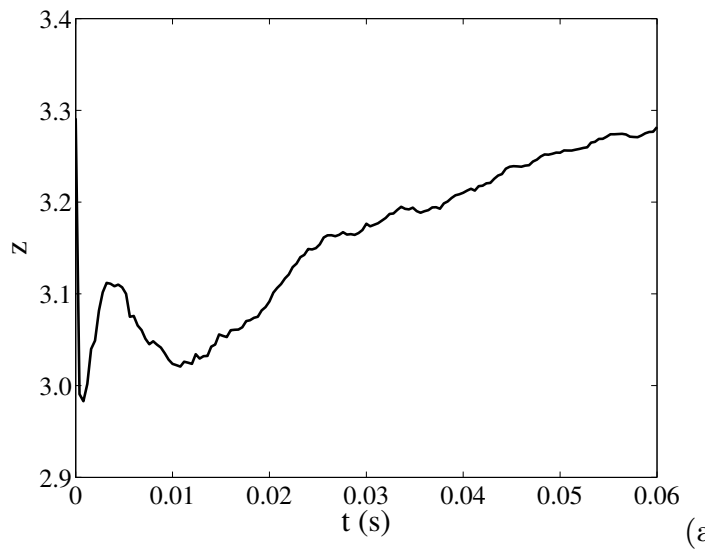

(a)

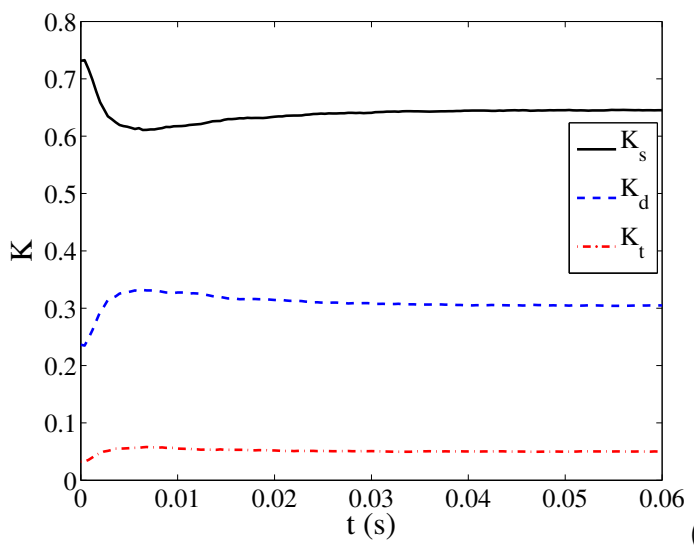

(b)

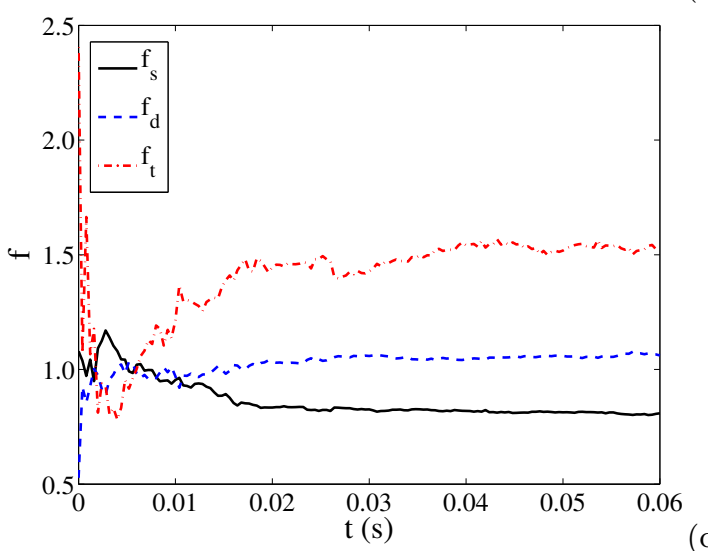

FIG. 16: Evolution of the coordination number (a), proportions of simple, double and tripe contacts (b) and mean force carried by each type of contact (c) with time during settlement for $\eta=2.5$. The forces are normalized by the mean force of all contacts.

by different types of contact follow the same trend. This evolution naturally underlies the growing aptitude of the packing to support larger stresses.

The analysis of the fabric may be pushed further to include the contact and force anisotropies, which underly the shear strength of granular materials [59-62]. These aspects and the local stresses in our simulated packings with frictional walls will be reported elsewhere. In fact, the evolution of contact types and their role in force

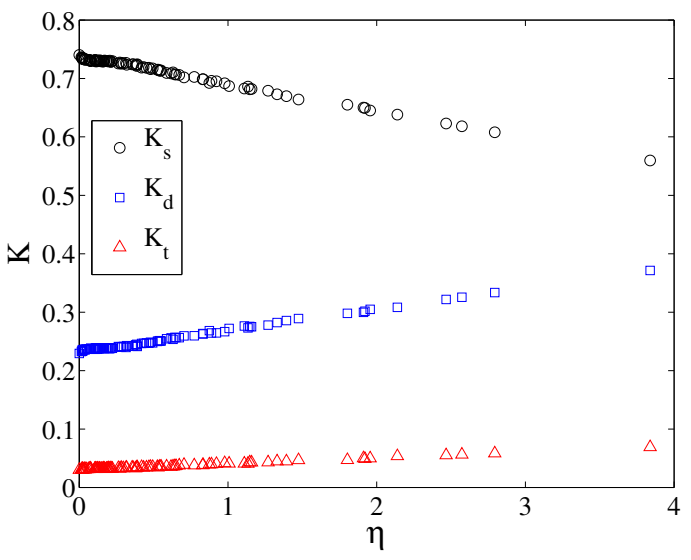

FIG. 17: Proportions of simple, double and triple contacts as a function of stress ratio.

transmission as presented above are the most intuitive features of the evolution of packings of polyhedral particles. But it can be shown that their effect is to reinforce the force anisotropy and more specifically the nearly rigid columns that are formed at the center of the packings as those observed in Fig. 8.

\section{CONCLUDING REMARKS}

In this paper, we analyzed the transient deformations of a granular material composed of polyhedral particles confined between two frictional walls, under the action of a constant vertical force applied in a single step. The polyhedral shape of the particles is representative of angular particles often found in nature and civil engineering applications. The friction at the top and bottom walls is also a realistic feature of granular fills used to sustain large external loads. In practice, the stability and settlement of granular materials under variable loading conditions cannot be predicted with confidence from classical elasto-plastic models of soil behavior based on a meanfield approximation of the kinematics and stresses. The role of the microstructural details, when a low number of particles is involved, is fundamental in the application of such models. The effect of dynamics when the load is not increased in a quasi-static fashion, as often assumed in models, is a fundamental concern for such systems. Finally, the stress gradients induced by wall friction complicate considerably the analysis since the wall friction and internal friction are intricately coupled. It is also obvious that the deformations are controlled to a large extent by the present state of the material and it is important to account carefully for the loading history.

For all these reasons, a quantitative description of transient deformations even in a model system with controlled geometry and material parameters appears to be a challenging task. The primary aim of this work was to show the feasibility of such a description by appropriate 
simulations and analysis. The sample-to-sample fluctuations led us to perform a large number of simulations with independent configurations. In order to ensure a reasonable reproducibility of the average settlements, we also added a preloading step to the simulations.

The results presented in this paper seem to indicate that the average deformation has a well-defined dependence with respect to the applied stress. But we also showed that the fluctuations, arising from the microstructural disorder varying among independent configurations, are large and scale with the average deformation. An- other important feature investigated in detail was the dependence of transient deformations on the aspect ratio as a result of the frictional feedback at the boundaries. This effect is captured by a simple model based on stress analysis and suggests that the increment of the aspect ratio is a relevant strain variable for a system with frictional walls. It shows that the settlement caused by a vertical load applied on a granular material does not depend only on the horizontal pressures acting on the material but also crucially on the surface area and friction coefficient of the support.
[1] H. M. Jaeger, S. R. Nagel, and R. P. Behringer, Reviews of Modern Physics 68, 1259 (1996).

[2] P. Cerasi and P. Mills, Phys. Rev. E 58, 6051 (1998).

[3] M. Foster, R. Fell, and M. Spannagle, Canadian Geotechnical Journal 37, 1000 (2000).

[4] S. Lobo-Guerrero and L. E. Vallejo, Granular Matter 8, 195 (2006).

[5] E. Tutumluer, H. Huang, Y. Hashash, and J. Ghaboussi, in AREMA, annula Conference (2006).

[6] P. Lade, Computers and Geotechnics 16, 123 (1994), ISSN 0266-352X, URL http://www.sciencedirect. com/science/article/pii/0266352X94900183.

[7] R. Nova, Journal of Mechanical behavior of Materials 5, 193 (1994), URL http://dx.doi.org/10.1063/1. 3179850.

[8] F. Darve and F. Laouafa, Mechanics of cohesive-frictional materials 5, 627 (2000).

[9] F. Nicot, F. Darve, and H. Dat Vu Khoa, Int. J. Numer. Anal. Meth. Geomech. 31, 1007 (2007), ISSN 1096-9853, URL http://dx.doi.org/10.1002/nag. 573.

[10] Radjai, Evesque, Bideau, and Roux, Phys. Rev. E 52, 5555 (1995).

[11] D. W. Howell, R. P. Behringer, and C. T. Veje, Chaos 9, 559 (1999), URL http://dx.doi.org/10.1063/1. 166430.

[12] Combe and Roux, Phys Rev Lett 85, 3628 (2000).

[13] F. Radjai and S. Roux, Phys. Rev. Lett. 89, 064302 (2002).

[14] F. Radjai and S. Roux, The Physics of Granular Media (Wiley-Vch, Berlin, 2004), chap. Contact dynamics study of $2 \mathrm{D}$ granular media : Critical states and relevant internal variables, pp. 165-186.

[15] D. Wood, Soil behaviour and critical state soil mechanics (Cambridge University Press, Cambridge, England, 1990).

[16] T. Schanz and P. A. Vermeer, Geotechnique 46, 145 (1996).

[17] S. Roux and F. Radjai, in Mechanics for a New Millennium, edited by H. Aref and J. Philips (Kluwer Acad. Pub., Netherlands, 2001), pp. 181-196.

[18] I. Vardoulakis, M. Goldscheider, and G. Gudehus, Int. J. Numer. Anal. Meth. Geomech. 2, 99 (1978), ISSN 1096-9853, URL http://dx.doi.org/10.1002/ nag. 1610020203 .

[19] O. Pouliquen, Phys. Fluids 11, 542 (1999), URL http: //link.aip.org/link/?PHF/11/542/1.

[20] GDR-MiDi, Eur. Phys. J. E 14, 341 (2004).
[21] G. H. Ristow and H. J. Herrmann, Physica A 213, 474 (1995).

[22] G. Ovarlez, C. Fond, and E. Clément, Phys. Rev. E 67, 060302 (2003).

[23] K. Braeuer, M. Pfitzner, D. O. Krimer, M. Mayer, Y. Jiang, and M. Liu, Phys. Rev. E 74, 061311 (2006).

[24] C. L. Gardner and D. G. Schaeffer, SIAM J. Appl. Math. 54, 1676 (1994).

[25] S. A. Watson, M. J. Adams, S. L. Rough, B. J. Briscoe, and T. Papathanasiou, in IUTAM Symposium on $\mathrm{Me}$ chanics of Granular and Porous Materials, edited by N. A. Fleck and A. C. E. Cocks (Kluwer Academic Publishers, 1997), pp. 415-426.

[26] S. Shukla, J. Loughran, and N. Sivakugan, Powder Technol. 192, 389 (2009).

[27] R. M. Nedderman, Statics and kinematics of granular materials (Cambr. Univ. Press, Cambridge, 1992).

[28] Radjai and Roux, Phys. Rev. E 51, 6177 (1995).

[29] S. C. du Pont, P. Gondret, B. Perrin, and M. Rabaud, Europhysics Letters 61, 492 (2003), ISSN 02955075, URL http://stacks.iop.org/0295-5075/61/i= $4 / a=492$.

[30] J. E. Maneval, K. M. Hill, B. E. Smith, A. Caprihan, and E. Fukushima, Granular Matter 7, 199 (2005), ISSN 1434-5021, URL http://dx.doi.org/10.1007/ s10035-005-0211-4.

[31] C. Zeilstra, J. Collignon, M. Hoef van der, N. Deen, and J. Kuipers, Powder Technology 184, 166 (2008), URL http://doc.utwente.nl/59740/.

[32] N. Taberlet, P. Richard, and R. Delannay, Computers \&amp; Mathematics with Applications 55, 230 (2008), ISSN 0898-1221, URL http://www. sciencedirect.com/ science/article/pii/S0898122107003495.

[33] M. Jean and J. J. Moreau, in Proceedings of Contact Mechanics International Symposium (Presses Polytechniques et Universitaires Romandes, Lausanne, Switzerland, 1992), pp. 31-48.

[34] J. Moreau, European Journal of Mechanics A/Solids supp., 93 (1994), formulation mathematiques titre du livre Contacts mechanics.

[35] F. Radjaï, in Physics of Dry Granular Media, edited by H. J. H. et al. (Kluwer Academic Publishers, Netherlands, 1998), pp. 305-312.

[36] M. Jean, Computer Methods in Applied Mechanic and Engineering 177, 235 (1999).

[37] F. Dubois and M. Jean, in Actes du sixième colloque national en calcul des structures - CSMA-AFM-LMS - 
(2003), vol. 1, pp. 111-118, URL https://subver.lmgc. univ-montp2.fr/trac_LMGC90v2/.

[38] F. Radjai and V. Richefeu, Mechanics of Materials 41, 715 (2009), ISSN 0167-6636, URL http://www.sciencedirect.com/science/article/ pii/S0167663609000386.

[39] N. V. Brilliantov, F. Spahn, J. M. Hertzsch, and T. Pöschel, Phys. Rev. E 53, 5382 (1996).

[40] T. Pöschel and V. Buchholtz, J. Phys. I France 5, 1431 (1995).

[41] H. J. Herrmann and S. Luding, Continuum Mechanics and Thermodynamics 10, 189 (1998).

[42] F. Radjai and F. Dubois, eds., Discrete-element Modeling of Granular Materials (Iste-Wiley, London, 2011).

[43] P. A. Cundall and O. D. L. Strack, Géotechnique 29, 47 (1979).

[44] J. J. Moreau, in CISM (1988), pp. 1-82.

[45] B. Brogliato, Nonsmooth mechanics (Springer, London, 1999).

[46] G. Saussine, Ph.D. thesis, Université Montpellier II (2004).

[47] E. Nezami, Y. Hashash, D. Zaho, and J. Ghaboussi, Computers and Geotechnics 31, 575 (2004).

[48] G. Saussine, C. Cholet, P. Gautier, F. Dubois, C. Bohatier, and J. Moreau, Comput. Methods Appl. Mech. Eng. 195, 2841 (2006).

[49] E. Azéma, F. Radjai, R. Peyroux, V. Richefeu, and G. Saussine, Eur. Phys. J. E 26, 327 (2008).

[50] E. Azéma, F. Radjai, and G. Saussine, Mechanics of Materials 41, 729 (2009), ISSN 0167-6636, URL http://www.sciencedirect.com/science/article/ pii/S0167663609000179.

[51] F. Radjai and V. Richefeu, Philosophical Transactions of the Royal Society A: Mathematical, Physical and Engineering Sciences 367, 5123 (2009), URL http://rsta.royalsocietypublishing.org/content/ 367/1909/5123. abstract.

[52] G. Ovarlez, E. Kolb, and E. Clément, Phys. Rev. E 64, 060302 (2001)

[53] M. Oda, J. Koshini, and S. Nemat-Nasser, Geotechnique 30, 479 (1980).

[54] M. M. Mehrabadi, S. Nemat-Nasser, H. M. Shodja, and G. Subhash, in Micromechanics of granular media (Elsevier, Amsterdam, 1988).

[55] B. Saint-Cyr, J.-Y. Delenne, C. Voivret, F. Radjai, and P. Sornay, Phys. Rev. E 84, 041302 (2011), URL http: //link.aps.org/doi/10.1103/PhysRevE.84.041302.

[56] E. Azéma and F. Radjai, Phys. Rev. E 85 031303 (2012), URL http://link.aps.org/doi/10. 1103/PhysRevE. 85.031303.

[57] F. Radjai, S. Roux, and J. J. Moreau, Chaos 9, 544 (1999), URL http://dx.doi.org/10.1063/1.166428.

[58] I. Agnolin and J.-N. Roux, Phys Rev E Stat Nonlin Soft Matter Phys 76, 061303 (2007).

[59] N. Kruyt and L. Rothenburg, Mechanics of Materials 36, 1157 (2004).

[60] S. J. Antony and M. R. Kuhn, International Journal of Solids and Structures 41 5863 (2004), URL http://www.sciencedirect. com/science/article/B6VJS-4CS4HW1-3/2/ aef50e5b64a4ca15f0c393ac7a4a9f80.

[61] E. Azéma, F. Radjai, R. Peyroux, and G. Saussine, Phys. Rev. E 76, 011301 (2007).

[62] C. Voivret, F. Radjai, J.-Y. Delenne, and M. S. E. Youssoufi, Phys. Rev. Lett. 102, 178001 (2009).

[63] The library was provided by the French Railway Company $\mathrm{SNCF}$. 